\title{
AN ENERGY UPGRADE OF THE REX-ISOLDE LINAC*
}

\author{
O. Kester, T. Sieber, H. Bongers, S. Emhofer, K. Rudolph, W. Carli, D. Habs \\ LMU München and the MLL München, 85748 Garching, Germany
}

\begin{abstract}
In 2002 the dominant experiment at CERN/ISOLDE was REX-ISOLDE. The LINAC delivered beams of radioactive isotopes at energies of $0.3,2$ and $2.2 \mathrm{MeV} / \mathrm{u}$ towards the two target stations. Due to the charge state breeder very different mass regions of the nuclear chart could be addressed from $\mathrm{Li}$ to $\mathrm{Sm}$. In order to perform Coulomb excitation and particle transfer experiments in the mass region up to $\mathrm{A}=150$, an energy of the LINAC is required, which is foreseen in two steps. The first step of the upgrade to $3.1 \mathrm{MeV} / \mathrm{u}$ will be done by using the MAFF $202.56 \mathrm{MHz}$ IH-cavity. The second step to energies close to $4.2 \mathrm{MeV} / \mathrm{u}$ will be a mayor change of the LINAC structure. There two 7-gap spiral resonators of REX-ISOLDE will be exchanged by a $202 \mathrm{MHz}$ IHcavity. Design calculations, cavity design and first measurements with the cavity dedicated for the first step of the upgrade will be presented.
\end{abstract}

\section{INTRODUCTION}

The radioactive beam experiment REX-ISOLDE at ISOLDE/CERN delivers post accelerated radioactive ion beams with a variable energy between 0.8 and $2.2 \mathrm{MeV} / \mathrm{u}$ [1]. In order to address many nuclear physics aspects, the full variety of beams available at ISOLDE should become accessible as accelerated beams for experiments. The method of charge state multiplication, which was introduced for the first time at REX-ISOLDE allows to access the different regions of the nuclear chart [2]. The charge-state breeder defines the time structure of the beam delivered to the LINAC and it adjusts the $\mathrm{A} / \mathrm{q}$ of the ions below 4.5. The higher charge states of the radioactive ions allow a moderate size of the accelerator.

In 2002 first experiments with radioactive nuclear beams have been carried out with REX-ISOLDE. Beams have been delivered to the $\gamma$-detector array MINIBALL and to small experiments in the second beam line at a maximum energy of $2.3 \mathrm{MeV} / \mathrm{u}$. In addition the commissioning of the LINAC has been performed in order to improve the beam quality and to reduce the beam energy spread [3]. It has been demonstrated that ions with masses up to $A=150$ could be accelerated with an overall efficiency of $2-3 \%$ from the ISOLDE target to the MINIBALL target. Until now the REX-ISOLDE LINAC could accelerate charge bred ions of ${ }^{23} \mathrm{Na}-{ }^{28} \mathrm{Na}$ and ${ }^{9} \mathrm{Li}$ to $2.3 \mathrm{MeV} / \mathrm{u}$ with a transmission through the LINAC of about $75 \%$. In the beam time with ${ }^{30} \mathrm{Mg}$ the beam could be delivered with smaller energy spread at $2.2 \mathrm{MeV} / \mathrm{u}$ and could be focussed through the target aperture of $5 \mathrm{~mm}$. Slightly lowering of the final energy and adjustment of the 7-gap cavities 2 and 3 to negative synchronous phases

*supported by the BMBF and the DFG the best longitudinal beam quality could be reached so far. For the Lithium run the second beam line was installed in the target area. With the successful acceleration of ${ }^{9} \mathrm{Li}^{2+}$ to $2.3 \mathrm{MeV} / \mathrm{u}$, REX-ISOLDE proved for the first time operation at the maximum $\mathrm{A} / \mathrm{q}$ of 4.5 above design energy.

Towards the energy upgrade of REX-ISOLDE and the planned acceleration of isotopes in the mass range of Uranium fission products, measurements have been taken to test the breeding efficiency of the EBIS in the vicinity of $\mathrm{A}=150$. Therefore, $\mathrm{a}{ }^{133} \mathrm{Cs}$ beam from the test source of REXTRAP was injected into the EBIS. The acceleration tests performed so far had to be been done with ${ }^{133} \mathrm{Cs}^{32+}$ $(\mathrm{A} / \mathrm{q}=4.16)$ at a breeding efficiency of about $1 \%$. Earlier measurements with Cs have shown a maximum charge state at $32+$ for a breeding time of $160 \mathrm{~ms}$.

\section{THE ENERGY UPGRADE}

To use the full range of isotopes from ISOLDE for nuclear physics experiments with Coulomb excitation and transfer reactions, higher beam energies are required. An increased energy of approx. $3 \mathrm{MeV} / \mathrm{u}$ allows studies of nuclear reactions up to mass $\mathrm{A}=85$ on deuterium targets. A beam energy of $4.2 \mathrm{MeV} / \mathrm{u}$ would be suitable up to mass $A=145$. Therefore an energy upgrade of the REXISOLDE LINAC to increase the maximum particle energy at the target has been started. In two steps it is foreseen to raise the beam energy to approx. $4.2 \mathrm{MeV} / \mathrm{u}$ by maintaining the beam quality.

The structure of the REX-ISOLDE LINAC for the two energy upgrade scenarios is shown in fig.1. In order to reach $3.1 \mathrm{MeV} / \mathrm{u}$ the simplest solution is to include a 9gap IH-cavity operating with $202.56 \mathrm{MHz}$ resonance frequency. Due to the delay of the final permission to run FRM II, it was proposed to modify the prototype of the Munich accelerator for Fission Fragments (MAFF) IH-7Gap resonator into such an IH-9-Gap cavity [4]. A major change of the LINAC structure will be required in order to reach energies of approximately $4.2 \mathrm{MeV} / \mathrm{u}$. Thus two of the 7-gap spiral resonators with 101.28 MHz resonance frequency have to be replaced by a $1.5 \mathrm{~m} \mathrm{IH}$-cavity with 202.56 $\mathrm{MHz}$ resonance frequency to boost the energy to required region of $3.75 \mathrm{MeV} / \mathrm{u}$. Then a 7 -gap $\mathrm{IH}$ resonator of the MAFF type cavity can be used to accelerate to final energies above $4 \mathrm{MeV} / \mathrm{u}$. The rfparameter of the 7-gap set-up and the 9-gap set-up are specified in table 1 .

\section{The MAFF IH-7gap resonator}

For the MAFF-LINAC an efficient 7-gap structure compared to the split ring resonators of REX-ISOLDE is required for the energy variation. Hence the 7-gap 


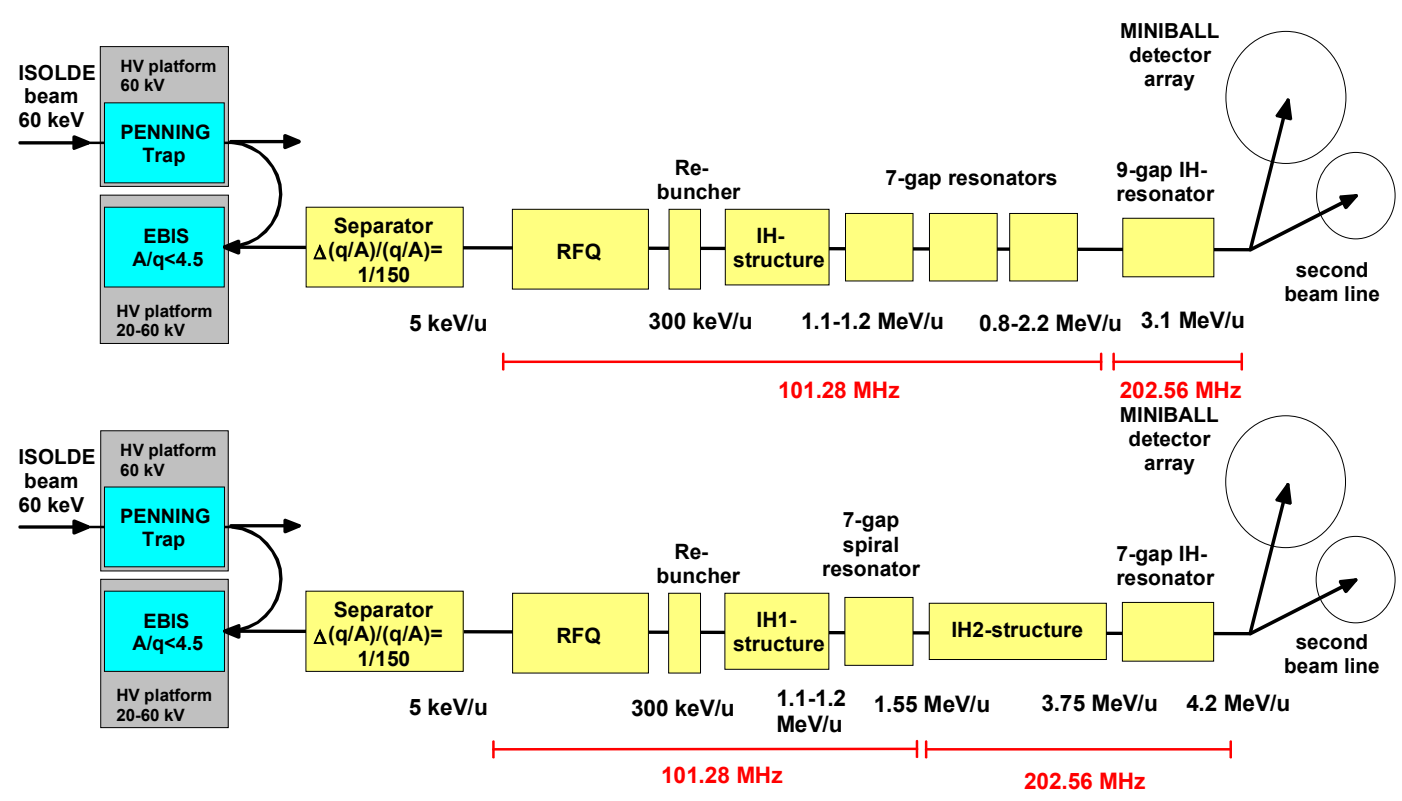

Figure 1: Structure of the REX-ISOLDE LINAC for the two upgrade scenarios. Several IH-structures with 202.56 $\mathrm{MHz}$ resonance frequency have to be included in order to raise the final beam energy.

structure has been designed as an IH-structure. Due to the higher shunt impedance of IH-structures a higher resonator voltage in combination with a very compact design can be achieved with the same rf-power compared to split ring resonators. Since the resonator is used both for acceleration and deceleration respectively, the cell length is kept constant. Based on the fact that the beam energy at MAFF will be varied between $3.7 \mathrm{MeV} / \mathrm{u}$ and $5.9 \mathrm{MeV} / \mathrm{u}$ a cell length of $74 \mathrm{~mm}$ was chosen, which corresponds to a design speed of $\beta=0.1$. This results in an total length of $518 \mathrm{~mm}$ for the seven cells. The inner tank length therefore is $520 \mathrm{~mm}$ and the overall outside length is $646 \mathrm{~mm}$. Low level measurements have been carried out to determine Q-values and shunt impedance (table 1) of both set-ups.

Table 1: Specification of the 7- and 9-gap IH-cavity

\begin{tabular}{|c|c|c|}
\hline & 7-gap & 9gap \\
\hline cell length [mm] & 74 & 55 \\
\hline gap length [mm] & 24 & $22-26$ \\
\hline drift tube length [mm] & 50 & 32 \\
\hline drift tube diameter [mm] & $20 / 26$ & $16 / 22$ \\
\hline max. A/q & 6.3 & 4.5 \\
\hline synchronous particle $\beta$ & 0.1 & 0.073 \\
\hline $\begin{array}{c}\text { shunt impedance }[\mathrm{M} \Omega / \mathrm{m}] \\
\text { (low level measurements) }\end{array}$ & 129 & 218 \\
\hline Q (measured) & 9800 & 10100 \\
\hline
\end{tabular}

Fig. 2 shows the power resonator tank in the 7-gap setup ready for beam tests at the MLL tandem. On a high energy beam line of the MLL a test bench for high power and beam measurements was installed. It was possible to obtain momentum spectra of a dc beam at different amplifier power and spectra for beam pulses at different phases, using a $70^{\circ}$ bending magnet positioned behind the resonator tank. The spectra with a dc-beam from the tandem were used to determine the effective shunt impedance. The measurement with pulsed beam injected into the 7-gap structures have been done to determine the ability to vary the beam energy by tuning the resonator's phase and amplitude.

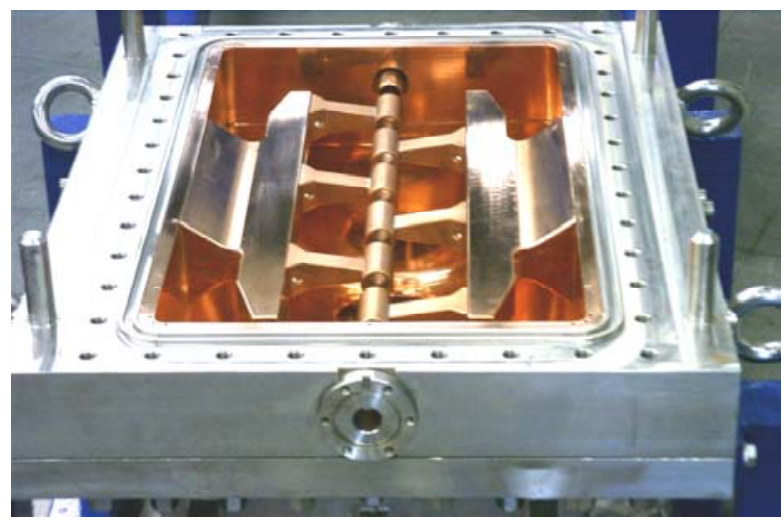

Figure 2: The resonator of the $202.56 \mathrm{MHz} \mathrm{IH}$ cavity.

Since the tandem can not deliver ions with $\mathrm{A} / \mathrm{q}=6.3$ and an energy of $4.15 \mathrm{MeV} / \mathrm{u}$ or $5.40 \mathrm{MeV} / \mathrm{u}$ respectively, a $66.4 \mathrm{MeV}{ }^{16} \mathrm{O}^{5+}$ beam was used $(\mathrm{A} / \mathrm{q}=3.2)$. Hence the rf-power could be reduced by a factor of 3.9. This way, the worst case scenario of deceleration and acceleration from $4.15 \mathrm{MeV} / \mathrm{u}$ could be examined. Fig. 3 shows the results of the power measurements. The effective shunt impedance remains constant although the calculated transit time factor (TTF) increases from 0.81 to 0.87 in the range of the rf-power level we have measured. Hence the absolute shunt impedance decreases, although the resonator's Q-value does not change significantly. Due to 
the acceleration far of the synchronous particle velocity, local changes of the gap voltage distribution lead to a drastic change in the TTF, which can compensate the expected increase of the TTF from calculations. However the requirements for MAFF of $82 \mathrm{M} \Omega / \mathrm{m}$ effective shunt impedance are exceeded by far.
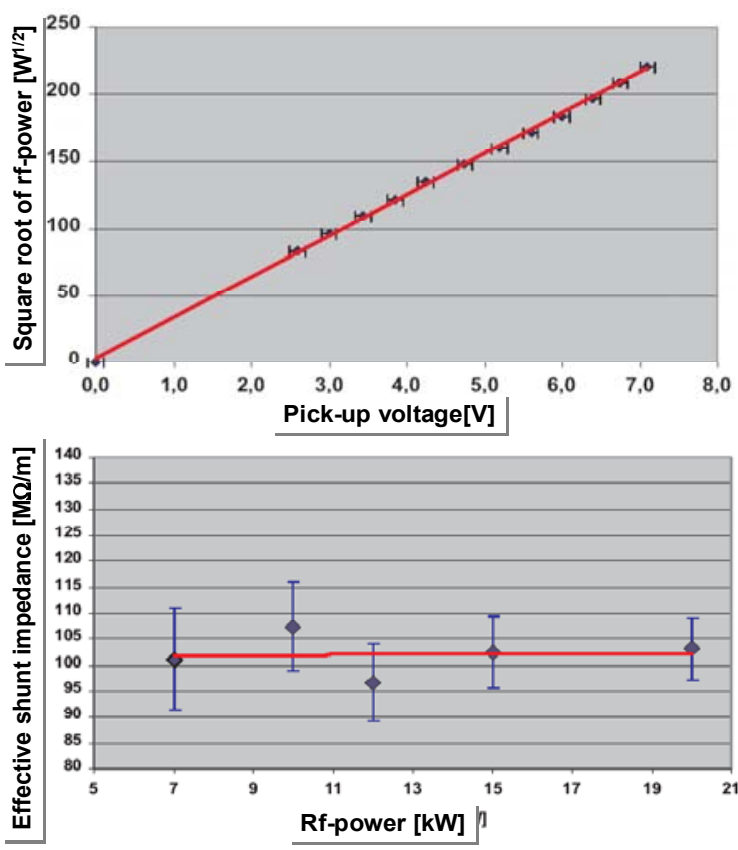

Figure 3: Measurements of the IH-7-gap resonator amplitude and shunt impedance.

\section{The IH-9-gap resonator}

The 9-gap set-up required for the first step energy upgrade has been examined with oxygen beams at the MLL test bench as well. A $\mathrm{O}^{5+}$-beam at 2.2, 2.25 and 2.3 $\mathrm{MeV} / \mathrm{u}$ from the Munich tandem was used to test the ability of the 9-gap IH-structure to post accelerate at power levels from 5 to $70 \mathrm{~kW}$. An energy spectrum is shown for $70 \mathrm{~kW}$ rf-power in fig.4. The tandem peak has been used for energy calibration. The drift tube structure was adjusted with constant cell length of $55 \mathrm{~mm}$.

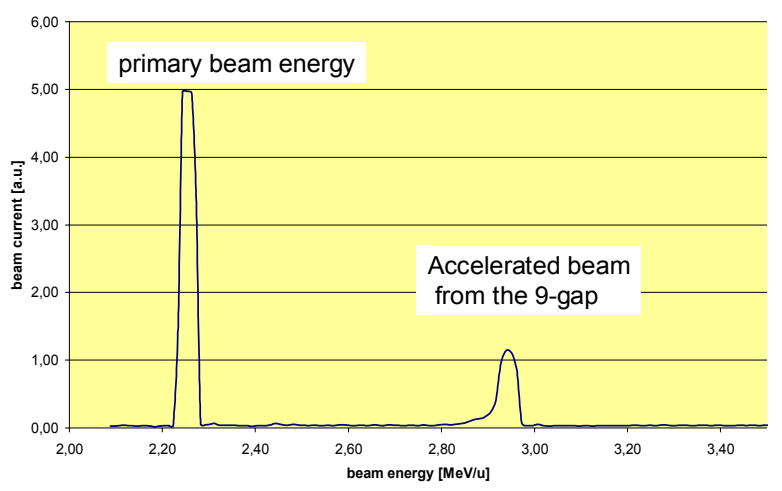

Figure 4: Adjustment of the gap voltage distribution.

The measured effective shunt impedance (fig.5) shows changes with the rf-power level, which corresponds to small values of the TTF below 0.8. This is a result of the cell length which was kept constant at $2.5 \mathrm{MeV} / \mathrm{u}$ synchronous particle energy. Therefore a higher injection energy led to higher effective shunt impedances shown in fig.5. As a result he drift tube structure has to be installed according to the velocity profile in case of the 9-gap setup, otherwise the required energy gain for REX-ISOLDE will not be possible.

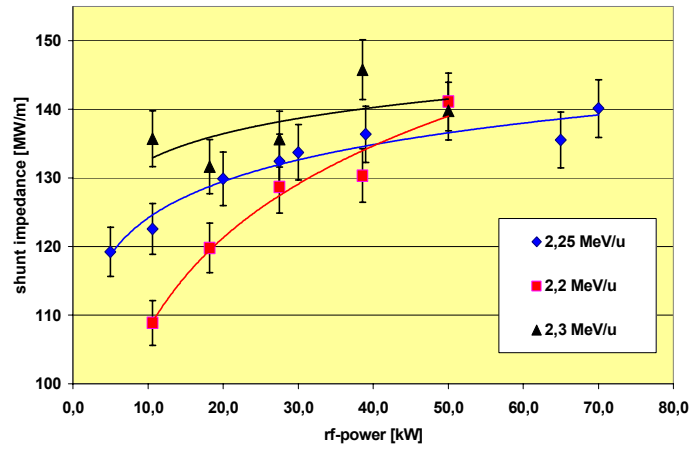

Figure 5: Effective shunt impedance of the 9-gap resonator for different injection energies.

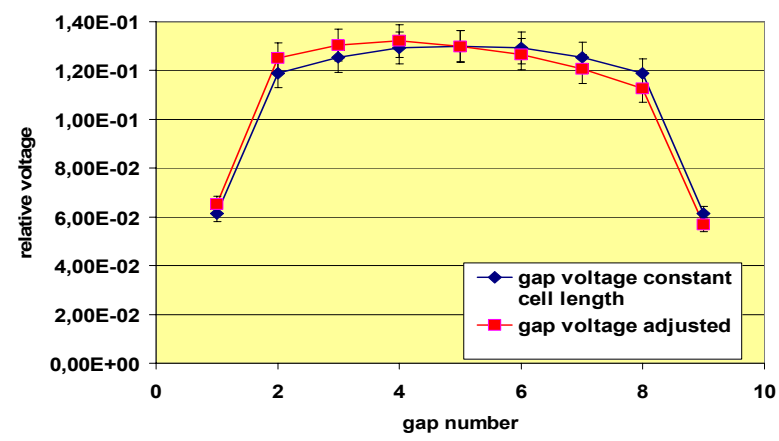

Figure 6: Adjustment of the gap voltage distribution.

With MAFIA the gap voltage distribution has been calculated for the constant cell length and the adjusted velocity profile. Taking that distribution shown in fig. 6 into account, beam dynamic calculations deliver a TTF of 0.87 and an effective shunt impedance of $165 \mathrm{M} \Omega / \mathrm{m}$, which is sufficient to reach $3 \mathrm{MeV} / \mathrm{u}$ at REX-ISOLDE with $90 \mathrm{~kW}$ rf-power.

\section{REFERENCES}

[1] O. Kester et al., "Accelerated radioactive beams from REX-ISOLDE", Proc. of EMIS14, Victoria, Canada, 2002, to be published in NIM

[2] O. Kester and D. Habs, "Charge breeding of intense radioactive beams", 8. Symp. on EBIS sources, AIP Conf. Proc. 572, p. 217

[3] S. Emhofer et al., "Beam measurements at the REXISOLDE LINAC", these proceedings

[4] O. Kester et al., "A short IH-cavity for the Energy Variation of Radioactive Ion Beams", Proc. of the EPAC2002, 3.-7. Juni, Paris, Frankreich, 2002. 